\title{
Supersymmetry searches in ATLAS
}

\author{
Federico Meloni*, on behalf of the ATLAS collaboration \\ Albert Einstein Center for Fundamental Physics, Laboratory for High Energy Physics, \\ University of Bern, Bern, Switzerland \\ E-mail: Federico.melonidcern.ch
}

This document summarises recent ATLAS results for searches for supersymmetric particles using LHC proton-proton collision data. Despite the absence of experimental evidence, weak scale supersymmetry remains one of the best motivated and studied Standard Model extensions. We consider both R-Parity conserving and R-Parity violating SUSY scenarios. The searches involve final states including jets, missing transverse momentum, light leptons, $\tau$ leptons or photons, as well as long-lived particle signatures. Sensitivity projections for the data that will be collected in 2015 are also presented.

The XXIII International Workshop on Deep Inelastic Scattering and Related Subjects April 27 - May 1, 2015

Southern Methodist University

Dallas, Texas 75275

${ }^{*}$ Speaker. 


\section{Introduction}

Supersymmetry (SUSY) provides an extension of the Standard Model (SM) by introducing partners of the known bosons and fermions differing by half-unit of spin. In the framework of an R-parity ${ }^{1}$ conserving minimal supersymmetric extension of the SM, SUSY particles are produced in pairs and the lightest supersymmetric particle (LSP) is stable and a possible candidate for dark matter. In many models, the LSP is the lightest neutralino $\tilde{\chi}_{1}^{0}$. Introducing non-zero R-parity violating (RPV) couplings into supersymmetric models provides additional phenomenology: the most relevant fact is the LSP is unstable and decays to SM particles.

SUSY can naturally solve the hierarchy problem by preventing a large fine-tuning in the Higgs sector: a typical natural SUSY spectrum contains light third generation squarks (stops and sbottoms), charginos and neutralinos. Such a spectrum is motivated by the fact that the dominant radiative corrections to the Higgs boson mass arise from the top quark in the SM. Thus, a relatively light stop can cancel out these corrections. Furthermore, the gaugino masses, related to the $\mu$ parameter in SUSY at tree level, are expected to be close to the electroweak scale.

This document reports several ATLAS[四] searches in the context of natural supersymmetry carried out using LHC proton proton collision data collected at $\sqrt{s}=8 \mathrm{TeV}$, and some early sensitivity projections for the first year of data taking at $\sqrt{s}=13 \mathrm{TeV}$. The ATLAS search strategy aims at covering every production mechanism, from strong to electro-weak, and final state composition (e.g. lepton multiplicity). The results will be presented following this same categorisation.

\section{Inclusive strong production searches}

The strong production of squarks and gluinos has been targeted with a comprehensive search strategy looking for events with an excess of missing transverse momentum $\left(E_{\mathrm{T}}^{\mathrm{miss}}\right)$ and different lepton multiplicities[[] [3]. For a single signal hypothesis, a combination of the results from different searches can provide stronger limits. These combinations rely on a detailed understanding of the correlations between the background estimates and the systematic uncertainties and provide the greatest benefit when the searches being combined have comparable sensitivity. We require the channels being combined to be mutually exclusive.

The results of searches in final states with jets, missing transverse momentum and either no or one isolated lepton are combined and interpreted in two simplified models where pair-produced squarks or gluinos decay through an intermediate chargino to the LSP [四]. No deviation from the SM expectation is observed. Limits are set in the $m(\tilde{g})-m\left(\tilde{\chi}_{1}^{0}\right)$ plane (Fig. $\mathbb{W}$ ) assuming $100 \%$ branching ratio for the considered decays of the SUSY particles. Gluino masses below $1.3 \mathrm{TeV}$ are excluded at $95 \%$ CL for a neutralino mass below $500 \mathrm{GeV}$. Pair produced squarks are excluded in an analogous model up to a mass of $850 \mathrm{GeV}$ at $95 \% \mathrm{CL}$ for massless neutralinos. These limits are an improvement of approximately $50 \mathrm{GeV}$ with respect to the results of the analyses before combination.

The discovery reach in the LHC Run-2 is expected to be greatly enhanced due to the increase in the LHC centre-of-mass collision energy. A study of the expected sensitivity to gluino pair

\footnotetext{
${ }^{1} \mathrm{R}$-parity is a discrete multiplicative symmetry defined as $R=-1^{3 B+L+2 s}$, where $B$ is the baryon number, $L$ the lepton number and $s$ the spin.
} 


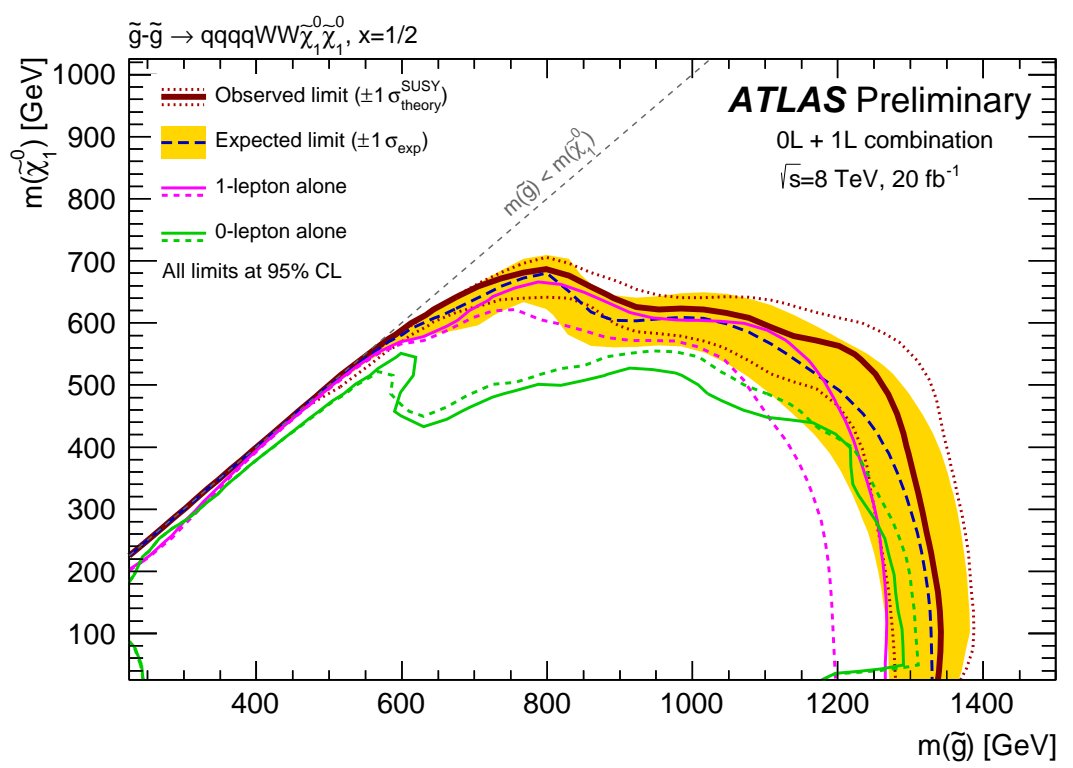

Figure 1: Observed and expected limits for simplified models of gluino-pair production with decays through an intermediate chargino, for chargino masses exactly half the mass difference between the gluino mass and the LSP mass. The yellow band includes all experimental uncertainties; the red dotted lines indicate the theory uncertainty on the cross-section. The individual limits from the 0-lepton analysis [[]] and the 1-lepton

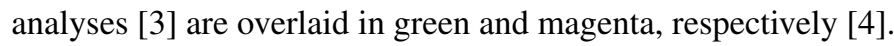

production with early $13 \mathrm{TeV}$ data has been performed [5]. A cut and count analysis has been optimized for different integrated luminosities, from 1 to $10 \mathrm{fb}^{-1}$. Signal regions are based on the event $E_{\mathrm{T}}^{\text {miss }}$ and effective mass, $m_{\text {eff }}$, defined as the scalar sum of transverse momenta of the jets and $E_{\mathrm{T}}^{\text {miss }}$. Figure $\square$ shows the expected sensitivities for two different assumptions on the uncertainties on the SM background determination: a discovery significance corresponding to more than 3 standard deviations can be achieved in the first year of data taking for the most favourable models.
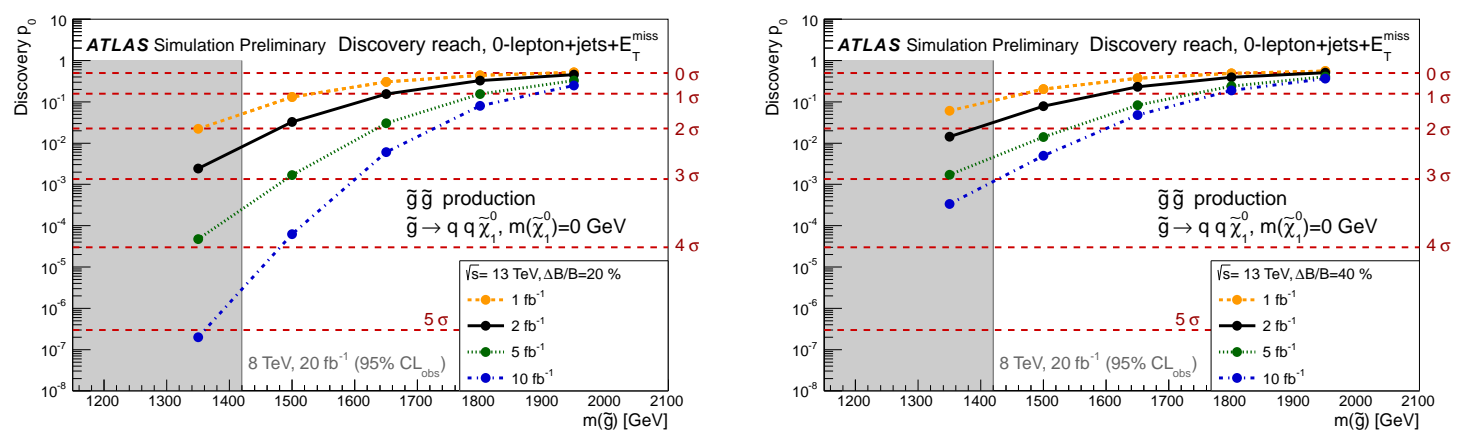

Figure 2: Discovery p0 values as a function of the gluino mass in the gluino-pair production models at a given integrated luminosity of $1,2,5$, or $10 \mathrm{fb}^{-1}$ (from inner to outer lines) with a hypothesis of $20 \%$ (left) or $40 \%$ (right) uncertainties on the total background prediction, respectively. For each integrated luminosity scenario, one baseline signal region is optimised for one target signal point with a hypothesis of $20 \%$ uncertainties on the total background prediction. These signal regions are used in both plots [[]]. 


\section{Third generation squark searches}

Final states from the decay of third generation squarks are typically extremely rich: they usually include leptons, multiple jets (possibly b-tagged) and $E_{\mathrm{T}}^{\mathrm{miss}}$. ATLAS carried out a set of analyses covering many complementary search channels. If the top squark is the next-to-lightest SUSY particle (NLSP), it may decay via several mechanisms: it can decay to a top quark and a neutralino, to a bottom quark, an on or off shell $\mathrm{W}$ boson, and a neutralino or finally via loop-suppressed diagrams to a charm quark and a neutralino. Figure [] shows the summary of the searches for these decay modes.

The top squark is excluded up to a mass of $700 \mathrm{GeV}$ for neutralino masses of up to $250 \mathrm{GeV}$, with some notable exceptions due to very challenging kinematic regimes represented by the dotted diagonal lines in the figure. These are compressed mass spectra that yield either soft decay products or final states similar to SM processes. One notable example is a top squark with a mass close to the sum of the W-boson and the neutralino masses: this signature looks like W-boson pair production.

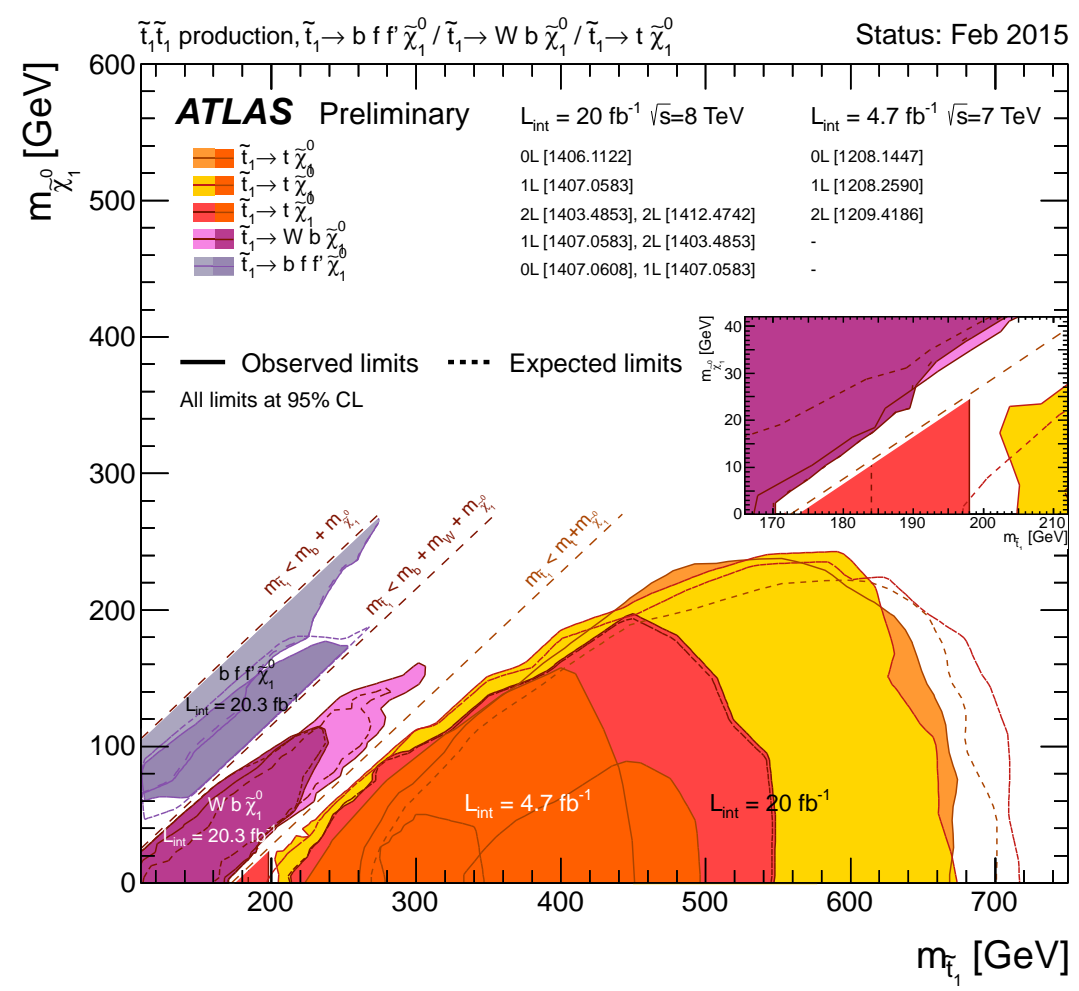

Figure 3: Summary of the dedicated ATLAS searches for top squark (stop) pair production based on up to $20.7 \mathrm{fb}^{-1}$ of pp collision data taken at $\sqrt{s}=8 \mathrm{TeV}$ and $4.7 \mathrm{fb}^{-1}$ of pp collision data taken at $\sqrt{s}=7$ TeV. Exclusion limits at $95 \% \mathrm{CL}$ are shown in the $\tilde{t}_{1}-\tilde{\chi}_{1}^{0}$ mass plane. The dashed and solid lines show the expected and observed limits, respectively, including all uncertainties except the theoretical signal cross section uncertainty (PDF and scale). The dotted lines represent the results obtained when reducing the nominal signal cross section by $1 \sigma$ of its theoretical uncertainty. Two decay modes are considered separately with $100 \%$ branching ratio: $\tilde{t}_{1} \rightarrow \tilde{\chi}_{1}^{0}$, where the $\tilde{t}_{1}$ is mostly $\tilde{t}_{R}$ and $\tilde{t}_{1} \rightarrow b+\tilde{\chi}_{1}^{ \pm}, \tilde{\chi}_{1}^{ \pm} \rightarrow W^{(*)}+\tilde{\chi}_{1}^{0}$. In the latter case, several hypotheses on the $\tilde{\chi}_{1}, \tilde{\chi}_{1}^{ \pm}, \tilde{\chi}_{1}^{0}$ mass hierarchy are assumed: fixed $\tilde{\chi}_{1}^{ \pm}$mass, $m\left(\tilde{\chi}_{1}^{ \pm}\right) \sim 2 \times m\left(\tilde{\chi}_{1}^{0}\right)$, fixed $m\left(\tilde{t}_{1}\right)-m\left(\tilde{\chi}_{1}^{ \pm}\right)=10 \mathrm{GeV}$ and fixed $m\left(\tilde{\chi}_{1}^{ \pm}\right)-m\left(\tilde{\chi}_{1}^{0}\right)=5 \mathrm{GeV}$. 
The measurement of spin correlations in $t \bar{t}$ events adds sensitivity in the case where the stop mass is close to the sum of the masses of the top quark and the neutralino[目]. This analysis is performed using di-leptonic $t \bar{t}$ events, requiring two leptons, at least two jets (at least one b-tag) and moderate $E_{\mathrm{T}}^{\text {miss }}$. The distribution of the azimuthal angle between the two leptons is the main discriminant variable for this search, which results in an exclusion of top squark masses between $177 \mathrm{GeV}$ and $191 \mathrm{GeV}$ for a neutralino with a mass of $1 \mathrm{GeV}$.

\section{Electroweak searches}

Direct production of pairs of charginos and neutralinos may be dominant if gluinos and squarks are heavier than a few $\mathrm{TeV}$. The searches for electroweak production of gauginos generally involve multi-lepton final states with small or no hadronic activity [] [ [8]. No excesses are found in data and limits are derived in simplified models for different assumptions on the decay branching ratio of the charginos and neutralinos. If one takes into account a decay via sleptons, a chargino with a mass of $700 \mathrm{GeV}$ can be excluded for a massless neutralino; the limit worsens significantly, going to $345 \mathrm{GeV}$, if the decay via SM gauge boson is taken into account instead.

An additional handle in such searches is the now known SM Higgs mass. Neutralinos and charginos are predicted to decay to a Higgs boson or to vector bosons over large regions of the SUSY phase space. Several decay chains have been investigated[0]: assuming a mass degenerate chargino-neutralino associated production, a Higgs boson may appear in the decay chain in association with a $\mathrm{W}$ boson and neutralinos that escape the detector. The Higgs decay branching fractions are exploited by combining mutually exclusive searches selecting final states with a leptonic W decay and either two b-jets, two photons or two or more additional leptons. Figure $\mathbb{\theta}$ shows the 95\% CL upper limit on the cross section of such models as a function of the NLSP mass under the assumption of a massless neutralino. Both the combined result, excluding a NLSP with a mass below $250 \mathrm{GeV}$, and the contributions by each analysis channel are shown.

\section{Long Lived Particle searches}

Long-lived supersymmetric particles can exist, if the mass spectra are almost degenerate or in R-Parity violating models. If a particle has a lifetime of order a few ns, it can decay inside the tracking detector, producing a decay vertex away from the primary vertex of the proton-proton interaction. Two signatures are considered: a dilepton signature where the vertex is formed from two oppositely charged leptons and a multi-track signature where the displaced vertex contains at least five charged-particle tracks[ए]].

A search was carried out for muon-like particles that move slower than the speed of light[ए]]. Different strategies were used to measure the particles speed and energy ionization loss $(\mathrm{dE} / \mathrm{dx})$. The silicon pixel detectors are used to measure $\mathrm{dE} / \mathrm{dx}$. The calorimeters and muon detectors are used to provide a time-of-flight measurement. No excess is observed in any signal region, and $95 \%$ CL limits are placed on the mass of long lived particles in various SUSY models. A cross section upper limit of $\sim 1 \mathrm{fb}$ on the cross section of the produced charged SUSY particle was measured in the phase space considered. 


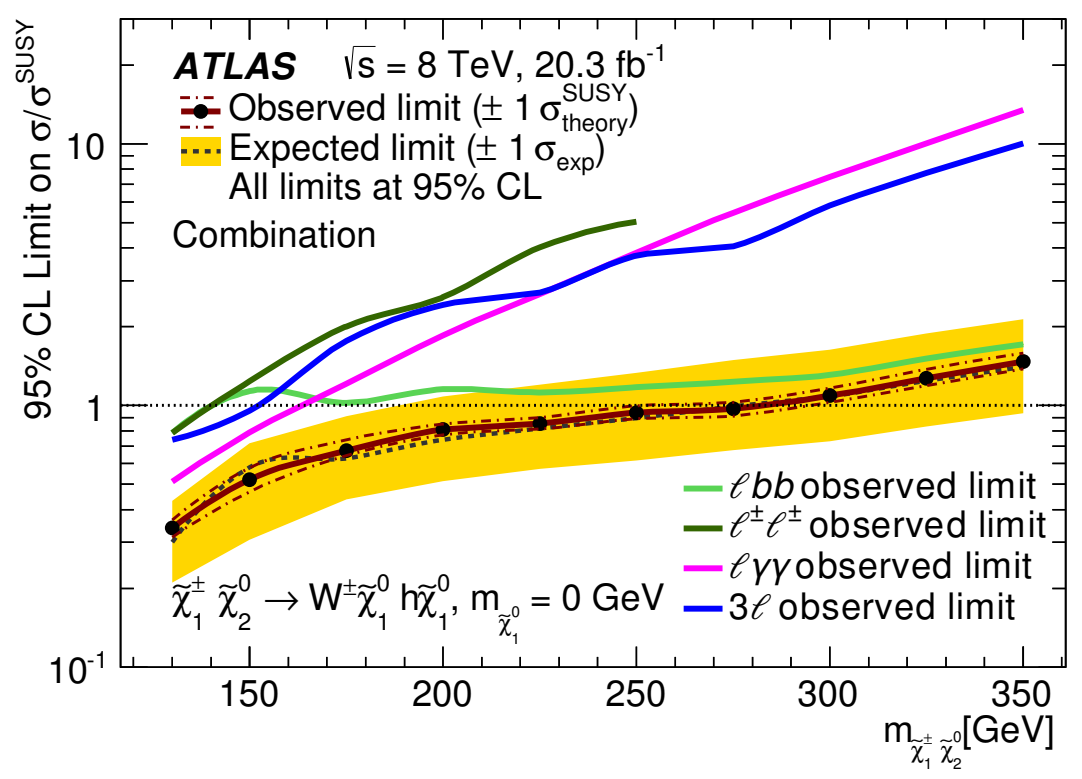

Figure 4: Observed (solid line) and expected (dashed line) 95\% CL upper limits on the cross section normalised by the simplified model prediction as a function of the common mass $\mathrm{m}\left(\chi_{1}^{ \pm}\right) \mathrm{m}\left(\chi_{2}^{0}\right)$ for $\mathrm{m}\left(\chi_{1}^{0}\right)=0$. The dash-dotted lines around the observed limit represent the results obtained when changing the nominal signal cross section up or down by the $\pm 1 \sigma_{\text {theory }}^{\text {SUSY }}$ theoretical uncertainty. The solid band around the expected limit represents the $\pm 1 \sigma_{\exp }$ uncertainty band where all uncertainties, except those on the signal cross sections, are considered.[Q]

The summary of the mentioned searches is shown in Fig. [ as upper limits on the gluino mass as a function of the average lifetime of the metastable SUSY particle, under the assumption of a neutralino LSP with a mass of $100 \mathrm{GeV}$.

\section{Conclusions}

ATLAS is conducting a comprehensive set of searches aiming at the natural SUSY models. Recent results using data recorded in 2012 in $\sqrt{s}=8 \mathrm{TeV}$ proton-proton collisions have been highlighted. No excess in data with respect to the SM expectation has been observed so far. However, much of the parameter space remains to be probed, and the search for SUSY at the LHC will continue. In particular, it will be pursued with the incoming Run-2 data with $13 \mathrm{TeV}$ centre-of-mass energy, which will bring new opportunities for discovery.

\section{References}

[1] ATLAS Collaboration, 2008 JINST 3 S08003.

[2] ATLAS Collaboration, JHEP 09 (2014) 176, arXiv: 1405.7875 [hep-ex].

[3] ATLAS Collaboration, JHEP 04 (2015) 116, arXiv: 1501.03555 [hep-ex].

[4] ATLAS Collaboration, ATLAS-CONF-2015-011, http://cds.cern.ch/record/2002555. 


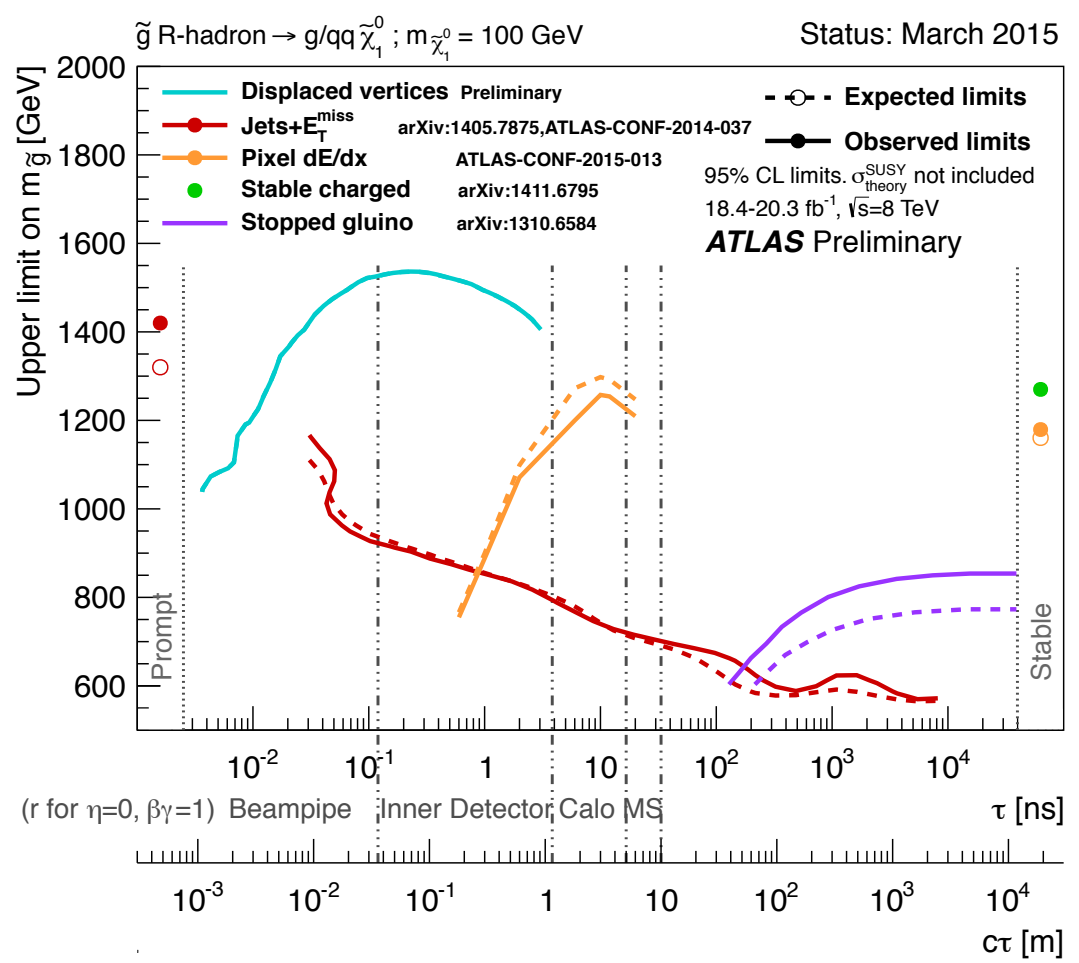

Figure 5: Constraints on the gluino mass-vs-lifetime plane for a split-supersymmetry model with the gluino R-hadron decaying into a gluon or light quarks and a neutralino with mass of $100 \mathrm{GeV}$. The solid lines indicate the observed limits, while the dashed lines indicate the expected limits. The area below the curves is excluded. For the displaced vertices result the expected and observed limits are identical. The analyses have sensitivity at lifetimes other than those shown, but only the limits at tested lifetimes are shown. The dots represent results for which the particle is assumed to be prompt or stable. In this context, stable means escaping the detector. [[2]]

[5] ATLAS Collaboration, ATL-PHYS-PUB-2015-005, http: //cds.cern.ch/record/2002608.

[6] ATLAS Collaboration, Phys. Rev. Lett. 114, 142001 (2015), arXiv: 1412.4742 [hep-ex].

[7] ATLAS Collaboration, JHEP 04 (2014)169, arXiv: 1402.7029 [hep-ex].

[8] ATLAS Collaboration, Phys. Rev. D 90, 052001 (2014), arXiv: 1405.5086 [hep-ex].

[9] ATLAS Collaboration, Eur. Phys. J. C (2015) 75:208, arXiv: 1501.07110 [hep-ex].

[10] ATLAS Collaboration, arXiv:1504.05162 [hep-ex].

[11] ATLAS Collaboration, JHEP 01 (2015) 068, arXiv:1411.6795 [hep-ex].

[12] https://twiki.cern.ch/twiki/bin/view/AtlasPublic/ supersymmetryPublicResults 